1 Hacettepe Journal of Mathematics and Statistics

h Volume 47 (3) (2018), 539-551

\title{
Hybrid genetic algorithms for global optimization problems
}

\author{
M. Asim*, W. Khan*†, O. Yeniay ${ }^{\ddagger}$, M. A. Jan*, N. Tairan ${ }^{\S}$, H. Hussian ${ }^{\star}$ and \\ Gai-Ge Wang"
}

\begin{abstract}
In the last two decades the field evolutionary computation has become a mainstream and several types of evolutionary algorithms are developed for solving optimization and search problems. Evolutionary algorithms (EAs) are mainly inspired from the biological process of evolution. They do not demand for any concrete information such as continuity or differentiability and other information related to the problems to be solved. Due to population based nature, EAs provide a set of solutions and share properties of adaptation through an iterative process. The steepest descent methods and Broyden-Fletcher-Goldfarb-Shanno (BFGS),Hill climbing local search are quite often used for exploitation purposes in order to improve the performance of the existing EAs. In this paper, We have employed the BFGS as an additional operator in the framework of Genetic Algorithm. The idea of add-in BFGS is to sharpen the search around local optima and to speeds up the search process of the suggested algorithm. We have used 24 benchmark functions which was designed for the special session of the 2005 IEEE-Congress on Evolutionary Computation (IEEE-CEC 06) to examine the performance of the suggested hybrid GA. The experimental results provided by HGBA are much competitive and promising as compared to the stand alone GA for dealing with most of the used test problems
\end{abstract}

Keywords: Global Optimization, Evolutionary Computation (EC), Evolutionary Algorithm (EA), Genetic Algorithm (GA), Hybrid GA.

Received : 07.10.2016 Accepted : 22.04.2017 Doi : 10.15672/HJMS.2017.473

\footnotetext{
*Department of Mathematics, Kohat University of Science \& Technology, Pakistan.

$\dagger$ Corresponding Author.

Email : mashwanigr8@gmail.com

${ }^{\ddagger}$ Hacettepe University Beytepe Mahallesi, 06800, Ankara, Turkey.

$\S$ College of Computer Science, King Khalid University Abha, Kingdom of Saudi Arabia.

TTechnical Education \& Vocational Training Authority, KPK, Pakistan.

"School of Computer Science and Technology, Jiangsu Normal University, China.
} 


\section{Introduction}

Global optimization has gained much attentions in both academia and industrial application over the past many years. In this regards, different test suites of optimization problems are designed in the existing literature of evolutionary computation. These problems are quite useful for thorough experimental computational testing and evaluation in order to design a powerful and robust optimization algorithm [1, 2]. The practical examples of optimization included the pooling/blending operations, heat exchanger network synthesis, phase and chemical reaction equilibrium, robust stability analysis, batch plant design under uncertainty, chemical reactor network synthesis, parameter estimation and data reconciliation, conformational problems in clusters of atoms and molecules, pump network synthesis, trim loss minimization, homogeneous azeotropic separation system, dynamic optimization problems in parameter estimation and in reactor network synthesis, and optimal control problems. In general, constrained minimization problem can be written as follows [3, 5]:

$$
\begin{aligned}
& \text { Minimize } f(x), x=\left(x_{1}, x_{2}, \ldots, x_{n}\right)^{T} \in R^{n} \\
& \text { Subject to }\left\{\begin{array}{l}
g_{i}(x) \leq 0, i=1,2, \ldots, p \\
h_{j}(x)=0, j=p+1, p+2, \ldots, q
\end{array}\right.
\end{aligned}
$$

where $x \in \Omega \subseteq S, \Omega$ is the feasible search space defined by $p$ number of inequality constraints, $q$ number of equality constraints and $S$ is the search space defined by parametric constraints: $L_{i} \leq x \leq U_{i}$. The inequality constraints that satisfy $g_{i}(\mathbf{x})=0$ are said to be an active constraints. It is important to be mentioned here that an equality constraints are always active. There are many types of optimization problems including multi-quadratic programming, bilinear and biconvex, generalized geometric programming, general constrained nonlinear optimization, bilevel optimization, complementarity, semi-definite programming, mixed-integer nonlinear optimization, combinatorial optimization and optimal control problems [3]. Generally all the above mentioned optimization problems can be categorized into two class including the constrained and unconstrained one. In this paper, we are interested in solving the unconstrained optimization problems with continuous variables. They are called boxed constrained optimization problems.

The last two decades are witnessed for the significant improvement and developments of optimization methods. Technically, optimization methods can be categorized into deterministic and stochastic ones. They have tackled diverse set of problems with continuous, discrete, integer, mixed integer variables [4]. The deterministic approaches are required the analytical properties of the problems while finding their optimal solutions [7]. The interval optimization [6], branch-and-bound [8, 9] and algebraic techniques [10], Simplex method [11], Hill climbing [12], Newton-Raphson method [13] are leading examples of the some deterministic approaches.

The stochastic approaches involve randomness to perform their search process. The simulated annealing [14], Monte Carlo sampling [15], stochastic tunneling [16], and parallel tempering [17], Genetic Algorithm (GA) [18], Evolutionary Strategies (ES) [19], Evolutionary Programming (EP) [20], Particle Swarm Optimization (PSO) [23], Ant Colony Optimization (ACO) [25] and differential evolution (DE) [26], Krill herd algorithms [35, 36, 37], Monarch butterfly optimization [38], Earthworm optimization algorithm [39], Plant propagation algorithm (PPA) [40, 41, 42, 43] are stochastic nature based optimization methods. Evolutionary computation is the collective name of these algorithms inspired by biological process of evolution, such as natural selection and genetic inheritance [44]. 
Hybrid evolutionary algorithms [45, 46, 51] have got much attention of the researchers and practitioners due to their high potentialities and capabilities in handling various real world problems and benchmark functions comprising high complexity, noisy environment, imprecision, uncertainty and vagueness [27, 28, 29, 47, 48, 49, 50].

In this paper, the suggested algorithm utilizes the Broyden-Fletcher-Goldfarb-Shanno (BFGS) algorithm [30,31] in combination with GA for population evolution at each multiple of $10^{t h}$ generations. The suggested algorithm is called HGBA have solved most of the test problems that were designed for the special session of the 2005 IEEE-congress on evolutionary computation (IEEE-CEC'05) [32]. HGBA have tackled most of the used test problems in an auspicious manner.

The rest of the paper is organized as follows. Section 2 presents the proposed algorithms. Section 3 demonstrates experimental results. Section 4 concludes this paper with some future plan.

\section{Hybridization of Genetic Algorithm with BFGS}

Genetic algorithm was first proposed by Holland inspired by the process of natural selection [33, 34]. GA is one of the most popular and well-known classical paradigms of evolutionary algorithms (EAs) [44]. This paradigm mainly relies on evolutionary operators such as mutation, crossover and selection to evolve their uniformly and randomly generated set of initial solutions called population. Due to population based nature, GA provides a set of optimal solutions in a single simulation run unlike traditional optimization methods. It simulates the survival of the fittest among the population over a consecutive generation. Since its inception [52, 53], several variants of GAs have been proposed and tackled different types of optimization and search problems, particularly in machine learning, scientific modeling, and artificial life and reviews a broad span of research, including the work of Mitchell and her colleagues [54].

The Local search algorithms like the GSAT and WalkSat, 2-opt algorithm, Iterated Local Search (ILS) perform search by applying local changes in the search space of solution to solution until stopping criteria is not satisfied. The combined use of efficient local search optimizers can speed up search process of the GA framework to locate the exact global optimum of the given optimization problems. The BFGS algorithm [30,31] is one of best well known hill-climbing local search method. Due to their fast convergence speed behavior, BFGS is applied to solve different nonlinear global optimization functions.

In the recent few years, several modifications have been made in the of the original framework of the genetic algorithm (GA) aiming at to alleviate their drawbacks. GA has successfully tackled different real-world problems such as space allocation problems on different sample test like warehouse, shelf, building floors and container and many others [55]. Different benchmark functions with continuous and discrete variables are also solved by GAs with great success. The combination of global and local searching (LS) can appeared in the form of hybrid evolutionary algorithms. They are quite useful for reducing the likelihood of the premature convergence which is normally occurred in the basic EAs for dealing with various search and optimization problems [56, 57, 62].

The suggested algorithm calls the BFGS [30,31] algorithm 2 at their algorithmic step 5 as explained in the algorithm 1, where the Hessian matrix of the BFGS algorithm is initialized with identity matrix and here after updated with gradient information of the current and previous iterations. 

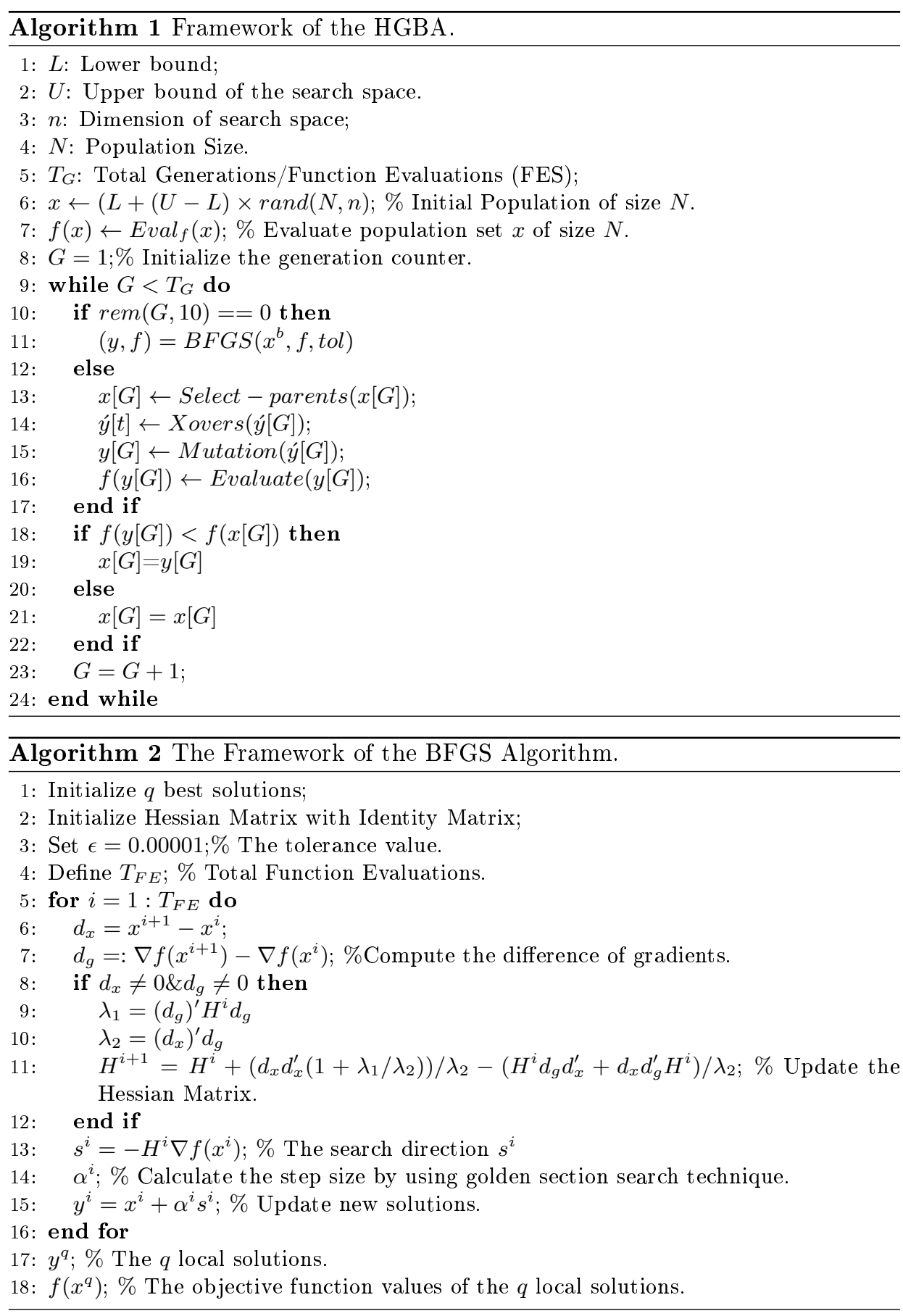
Table 1. Classification and properties of Tested functions

\begin{tabular}{|l|l|l|l|}
\hline Unimodel & Multimodel & Separable & Non Separable \\
\hline f01 & f06 & f01 & f02 \\
f02 & f07 & f09 & f03 \\
f04 & f08 & f15 & f04 \\
f05 & f10 & No & f05 \\
No & f11 & No & f06 \\
No & f12 & No & f07 \\
No & f13 & No & f10 \\
No & f14 & No & f11 \\
No & f15 & No & f12 \\
No & f16 & No & f13 \\
No & f17 & No & f14 \\
No & f18 & No & f16 \\
No & f19 & No & f17 \\
No & f20 & No & f18 \\
No & f21 & No & f19 \\
No & f22 & No & f20 \\
No & f23 & No & f21 \\
No & f24 & No & f22 \\
No & f25 & No & f23 \\
No & No & No & f24 \\
No & No & No & f25 \\
\hline
\end{tabular}

\section{Discussion on Experimental Results}

Continuous optimization problems have wide practical applications ranging from simple parameter identification in data-model fitting to intrinsic design-parameter optimization in complex technical systems in sciences and engineering fields. Different test suites of optimization with diverse characteristics as explained in the Table 1 are quite important for examining the overall performance of optimization algorithms in terms of convergence rate,precision and robustness.

In this paper, we have chosen test suite 25 benchmark functions [32] that comprising different characteristics. A brief summary regarding the used test functions denoted by $f_{1}-f_{25}$ is hereby summarized in the Table 1 .

The Table 1 provides the name of each test function, $f_{1}-f_{24}$ and its variables' value range is recorded as appears in the original Technical Report [32]. The dimension $\mathrm{N}$ of each solution vector used in the experiments is also recorded together with the fitness value of the optimal solution $\mathrm{f}\left(\mathrm{x}^{*}\right)$. The CEC'05 test functions are characterized as follows: the first five functions $f_{1}-f_{5}$ are unimodal and shifted; the second seven test functions $f_{6}-f_{12}$ are basic multimodal and shifted; the third two functions $f_{1} 3-f_{1} 4$ are expanded multimodal; and the fourth eleven functions $f_{15}-f_{25}$ are hybrid composition (i.e., all of them are non-separable, rotated, and multimodal functions containing a large number of local minima). Further details and evaluation criteria of the IEEE-CEC05 are given in [32].

We have carried out experiments at the platform:

- Operating system: Windows XP Professional.

- Programming language of the algorithms: Matlab. 
- CPU: Core 2 Quad 2.4 GHz.

- RAM: 4 GB DDR2 $1066 \mathrm{MHz}$.

- Execution: 30 times independent simulation of each algorithm with different random seeds.

Evolutionary Algorithms (EAs) are searching for the global optimum in their search space $R^{n}$ comprising $n$ dimensions. Initially, EAs require a set of $N$ solutions to evolve them user defined function evaluations (FES). In this paper, the experiments performed according to parameter settings described as follows:

- $N=100$, the population size.

- $n=2,5,10,20,30$ are dimensions of the search space.

- $F E S=n \times N$, total function evaluations.

- $q=5$, allowed best solutions for BFGS to works in the HGBA framework.

The algorithmic behavior of the proposed HGBA is verified by CEC05 problems with parameter settings as mentioned above. We have recorded all experimental results in minimum, mean, median, standard deviation and maximum values with different settings of $n=2,5,10,20, n=30$ while solving each test problem of the IEEE-CEC05 test suite [32]. It is important to mentioned here that we did not include all experimental results obtained with different settings of $n$.

Table 2 provides the numerical results of problems solved with $n=10$ dimension. Table 3 presents the objective function values each CEC'05 benchmark function with $n=30$ dimension. Both these tables clearly indicate that the suggested hybrid version of GA has solved most functions with better performance as compared to the basic GA on most problems.

The convergence graph of the HGBA versus GA are illustrated in the figures 1-1 for benchmark functions with search space dimension $n=10$ and $n=30$ in their 25 independent runs of simulation. The figure 1 shows the evolution of average function values within allowed function evaluations (FES). While the 1 demonstrates the average evolution in the objective function values of some CEC '05 test problems solved with search space dimension $n=30$. It can see from these figures, the convergence speed of the proposed algorithm is much better than the basic genetic algorithm (GA) while elapsing less function evaluations to reach near to the global optimum of the most CEC05 test problems.

\section{Conclusion}

Global optimization problems offer many challenges to evolutionary computing (EC) communities due to the existence of nonlinearity and multi-modality in their formulation structures. The stand-alone local search optimization methods are mostly unable to deal with such problems. Currently, hybridization of local search optimizers with existing meta-heuristic algorithms have got much attention of researchers in EC field. In this paper, a hybrid population-based global optimization algorithm is proposed that combines genetic algorithm (GA) with BFGS. The proposed algorithm denoted by HGBA are combined GA with BFGS in an ensemble manner to promote information sharing among the population and thus enhance the searching efficiency of basic GA. The performance of the proposed HGBA is evaluated on a diverse set of benchmark functions designed for the special session of the 2005 IEEE-CEC [32]. The experimental results show the proposed algorithm have performed better than GA in terms of better convergence speed near to the known optimal and hence not get stuck in local optimum of the most problems.

In future, we intend to analyze the impact of the proposed algorithm by employing some other effective local search optimizers and search operators such as improved variants differential evolution [26], particle swarm optimization (PSO) [23] and ant colony 
Table 2. The Numerical Results of the HGBA versus GA for each CEC'20O5 test problems with $n=10$ dimension [32].

\begin{tabular}{|c|c|c|c|c|}
\hline \multicolumn{5}{|c|}{ a)Hybrid Genetic Algorithm, b) Genetic Algorithm } \\
\hline CEC'05 Functions & Best & Mean & Std & Algorithm \\
\hline \multirow[b]{2}{*}{ f01 } & 0.000001 & 0.000001 & 0.521016 & $\mathrm{a}$ \\
\hline & 0.000000 & 0.000000 & 0.306146 & b \\
\hline \multirow[b]{2}{*}{ f02 } & 0.000000 & 0.000000 & 0.198374 & $\mathbf{a}$ \\
\hline & 0.000000 & 0.000000 & 0.445100 & $\mathrm{~b}$ \\
\hline \multirow[b]{2}{*}{ f03 } & 0.000000 & 0.000000 & 637.666968 & $\mathbf{a}$ \\
\hline & 0.000000 & 0.000105 & 14.057797 & $\mathrm{~b}$ \\
\hline \multirow[b]{2}{*}{ f04 } & 0.000000 & 0.000000 & 0.327159 & $\mathbf{a}$ \\
\hline & 0.000000 & 0.000000 & 0.039318 & $\mathrm{~b}$ \\
\hline \multirow[b]{2}{*}{ f05 } & 0.000000 & 0.000000 & 32.036672 & $\mathbf{a}$ \\
\hline & 0.000000 & 0.000002 & 40.692395 & $\mathrm{~b}$ \\
\hline \multirow[b]{2}{*}{ f06 } & 0.000000 & 0.000000 & 13.781630 & $\mathbf{a}$ \\
\hline & 0.000000 & 0.000283 & 10.536194 & $\mathrm{~b}$ \\
\hline \multirow[b]{2}{*}{ f07 } & 0.000009 & 3.557594 & 2.587509 & $\mathbf{a}$ \\
\hline & 0.004455 & 2.986633 & 1.413326 & $\mathrm{~b}$ \\
\hline \multirow[b]{2}{*}{ f08 } & 0.000000 & 0.000000 & 0.076302 & $\mathbf{a}$ \\
\hline & 0.000000 & 0.000000 & 0.197469 & $\mathrm{~b}$ \\
\hline \multirow[b]{2}{*}{ f09 } & 0.000000 & 0.000000 & 0.311911 & $\mathbf{a}$ \\
\hline & 0.000000 & 0.000000 & 0.426611 & $\mathrm{~b}$ \\
\hline \multirow[b]{2}{*}{ f10 } & 0.000061 & 0.007804 & 0.103723 & $\mathbf{a}$ \\
\hline & 0.004425 & 0.021671 & 0.120534 & $\mathrm{~b}$ \\
\hline \multirow[b]{2}{*}{ f11 } & 0.000000 & 0.000000 & 0.206919 & $\bar{a}$ \\
\hline & 0.000000 & 0.000000 & 2.123631 & $\mathrm{~b}$ \\
\hline \multirow[b]{2}{*}{ f12 } & 0.000000 & 0.000000 & 0.012976 & $\mathrm{a}$ \\
\hline & 0.000000 & 0.000000 & 0.005400 & b \\
\hline \multirow[b]{2}{*}{ f13 } & 0.000006 & 0.000768 & 0.040584 & $\mathbf{a}$ \\
\hline & 0.000024 & 0.000056 & 0.041487 & $\mathrm{~b}$ \\
\hline \multirow[b]{2}{*}{ f14 } & 1457.115466 & 1457.115806 & 4.142761 & $\mathbf{a}$ \\
\hline & 1457.215625 & 1457.259106 & 7.013462 & $\mathrm{~b}$ \\
\hline \multirow[b]{2}{*}{ f15 } & 1024.120243 & 1024.157612 & 22.599572 & $\mathbf{a}$ \\
\hline & 1024.994920 & 1025.288941 & 22.631336 & $\mathrm{~b}$ \\
\hline \multirow[b]{2}{*}{ f16 } & 1018.156033 & 1018.192758 & 14.263865 & $\mathbf{a}$ \\
\hline & 1018.836790 & 1019.096324 & 12.314532 & $\mathrm{~b}$ \\
\hline \multirow[b]{2}{*}{$\mathrm{f} 17$} & 827.203398 & 827.205103 & 19.246432 & $\mathbf{a}$ \\
\hline & 827.217635 & 832.500164 & 15.346813 & $\mathrm{~b}$ \\
\hline \multirow[b]{2}{*}{ f18 } & 1250.190437 & 1250.106352 & 6.944744 & $\mathbf{a}$ \\
\hline & 1250.705684 & 1251.048538 & 8.034050 & $\mathrm{~b}$ \\
\hline \multirow[b]{2}{*}{ f19 } & 885.666535 & 916.629538 & 67.349620 & $\mathbf{a}$ \\
\hline & 894.251157 & 921.019167 & 54.960124 & $\mathrm{~b}$ \\
\hline \multirow[b]{2}{*}{$\mathrm{f} 20$} & 1341.214724 & 1341.214724 & 0.000000 & $\mathrm{a}$ \\
\hline & 1341.214724 & 1341.214724 & 0.000000 & $\mathrm{~b}$ \\
\hline \multirow[b]{2}{*}{$\mathrm{f} 21$} & 1180.095532 & 1185.909739 & 16.297264 & $\mathbf{a}$ \\
\hline & 1188.596456 & 1236.814978 & 21.359977 & $\mathrm{~b}$ \\
\hline
\end{tabular}


Table 3. The Numerical Results of the HGBA versus GA for each CEC'20O5 test problems with $n=30$ dimension [32].

\begin{tabular}{|c|c|c|c|c|}
\hline \multicolumn{5}{|c|}{ a) Hybrid Genetic Algorithm, b) Genetic Algorithm. } \\
\hline CEC'05 Functions & Best & Mean & Std & Algorithm \\
\hline \multirow[b]{2}{*}{ f01 } & 0.000000 & 0.000000 & 605.382503 & $\mathbf{a}$ \\
\hline & 0.000000 & 0.000000 & 661.259167 & $\mathrm{~b}$ \\
\hline \multirow[b]{2}{*}{ f02 } & 0.000003 & 0.039364 & 942.982813 & $\mathbf{a}$ \\
\hline & 0.000119 & 0.330952 & 1124.284441 & GA \\
\hline \multirow[b]{2}{*}{ f03 } & 315225.984858 & 539100.620758 & 5887328.994022 & $\mathrm{a}$ \\
\hline & 112502.970129 & 425041.073907 & 1309109.898453 & b \\
\hline \multirow[b]{2}{*}{ f04 } & 0.000040 & 0.149584 & 1154.242033 & $\mathbf{a}$ \\
\hline & 1218.002421 & 9074.364177 & 12412.710359 & $\mathrm{~b}$ \\
\hline \multirow[b]{2}{*}{ f05 } & 0.000000 & 0.000003 & 1366.160967 & $\mathbf{a}$ \\
\hline & 0.000000 & 0.000333 & 1403.301756 & $\mathrm{~b}$ \\
\hline \multirow[b]{2}{*}{ f06 } & 1.231123 & 4.107561 & 47669318.286299 & $\mathbf{a}$ \\
\hline & 2.318733 & 4.922776 & 30969857.223058 & $\mathrm{~b}$ \\
\hline \multirow[b]{2}{*}{ f07 } & 20.267766 & 20.279503 & 0.015343 & $\mathrm{a}$ \\
\hline & 20.204322 & 20.277736 & 0.036564 & $\mathrm{~b}$ \\
\hline \multirow[b]{2}{*}{ f08 } & 0.000000 & 3.422413 & 10.595200 & $\mathbf{a}$ \\
\hline & 0.000026 & 5.327192 & 11.222839 & $\mathrm{~b}$ \\
\hline \multirow[b]{2}{*}{ f09 } & 17.676048 & 17.676048 & 8.873088 & $\mathrm{a}$ \\
\hline & 16.273877 & 18.097991 & 9.534268 & $\mathbf{b}$ \\
\hline \multirow[b]{2}{*}{ f10 } & 6.364467 & 7.146139 & 0.286591 & $\mathbf{a}$ \\
\hline & 7.327944 & 7.471782 & 0.455529 & $\mathrm{~b}$ \\
\hline \multirow[b]{2}{*}{ f11 } & 12.600003 & 40.466356 & 3909.201355 & $\mathbf{a}$ \\
\hline & 57.433299 & 553.051436 & 7246.718863 & $\mathrm{~b}$ \\
\hline \multirow[b]{2}{*}{ f12 } & 0.895147 & 1.349706 & 1.421402 & $\mathbf{a}$ \\
\hline & 1.018349 & 1.283563 & 1.315277 & $\mathrm{~b}$ \\
\hline \multirow[b]{2}{*}{$\mathrm{f} 13$} & 3.103262 & 3.103262 & 0.068194 & $\mathbf{a}$ \\
\hline & 3.366543 & 3.456506 & 0.074904 & $\mathrm{~b}$ \\
\hline \multirow[b]{2}{*}{ f14 } & 1360.491281 & 1360.554486 & 26.255421 & $\mathbf{a}$ \\
\hline & 1360.522848 & 1360.720281 & 27.040415 & $\mathrm{~b}$ \\
\hline \multirow[b]{2}{*}{ f15 } & 1287.077900 & 1297.099559 & 22.730716 & $\mathbf{a}$ \\
\hline & 1288.507390 & 1299.373887 & 23.507829 & $\mathrm{a}$ \\
\hline \multirow[b]{2}{*}{ f16 } & 1290.781525 & 1296.891223 & 20.723626 & $\mathbf{a}$ \\
\hline & 1293.072508 & 1298.831638 & 23.050568 & $\mathrm{~b}$ \\
\hline \multirow[b]{2}{*}{ f17 } & 1255.746668 & 1260.787053 & 14.183325 & $\mathbf{a}$ \\
\hline & 1258.658973 & 1259.159586 & 13.039324 & $\mathrm{~b}$ \\
\hline \multirow[b]{2}{*}{ f18 } & 1342.330076 & 1379.304890 & 29.825873 & $\mathrm{a}$ \\
\hline & 1317.106976 & 1382.449478 & 30.816738 & b \\
\hline \multirow[b]{2}{*}{ f19 } & 1153.289503 & 1211.524349 & 25.341379 & $\mathbf{a}$ \\
\hline & 1189.855128 & 1238.001648 & 23.684564 & $\mathrm{~b}$ \\
\hline \multirow[b]{2}{*}{$\mathrm{f} 20$} & 1391.379431 & 1400.300400 & 27.610007 & $\mathbf{a}$ \\
\hline & 1395.714142 & 1410.221171 & 20.295410 & $\mathrm{~b}$ \\
\hline \multirow[b]{2}{*}{$\mathrm{f} 21$} & 1356.522833 & 1365.801357 & 12.111518 & $\mathbf{a}$ \\
\hline & 1372.117983 & 1441.648192 & 25.123694 & $\mathrm{~b}$ \\
\hline
\end{tabular}

optimization algorithms [25] with combined self-adaptive procedures. We also our proposed HGBA to solve constrained test suites of the IEE-CEC series [63]. 

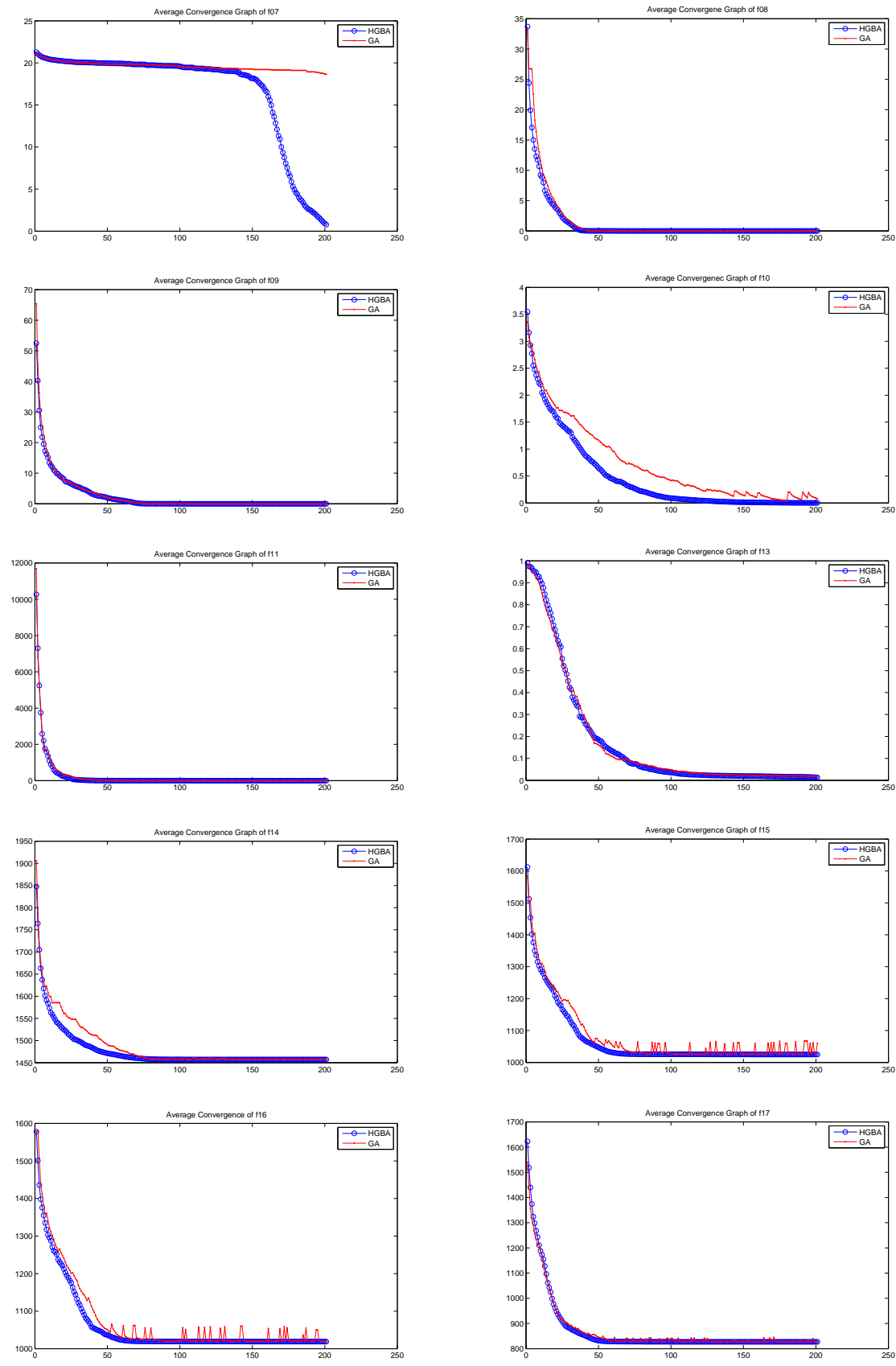

Figure 1. The convergence graph displayed by HGBA versus GA for CEC'05 [32] with ten variables. 

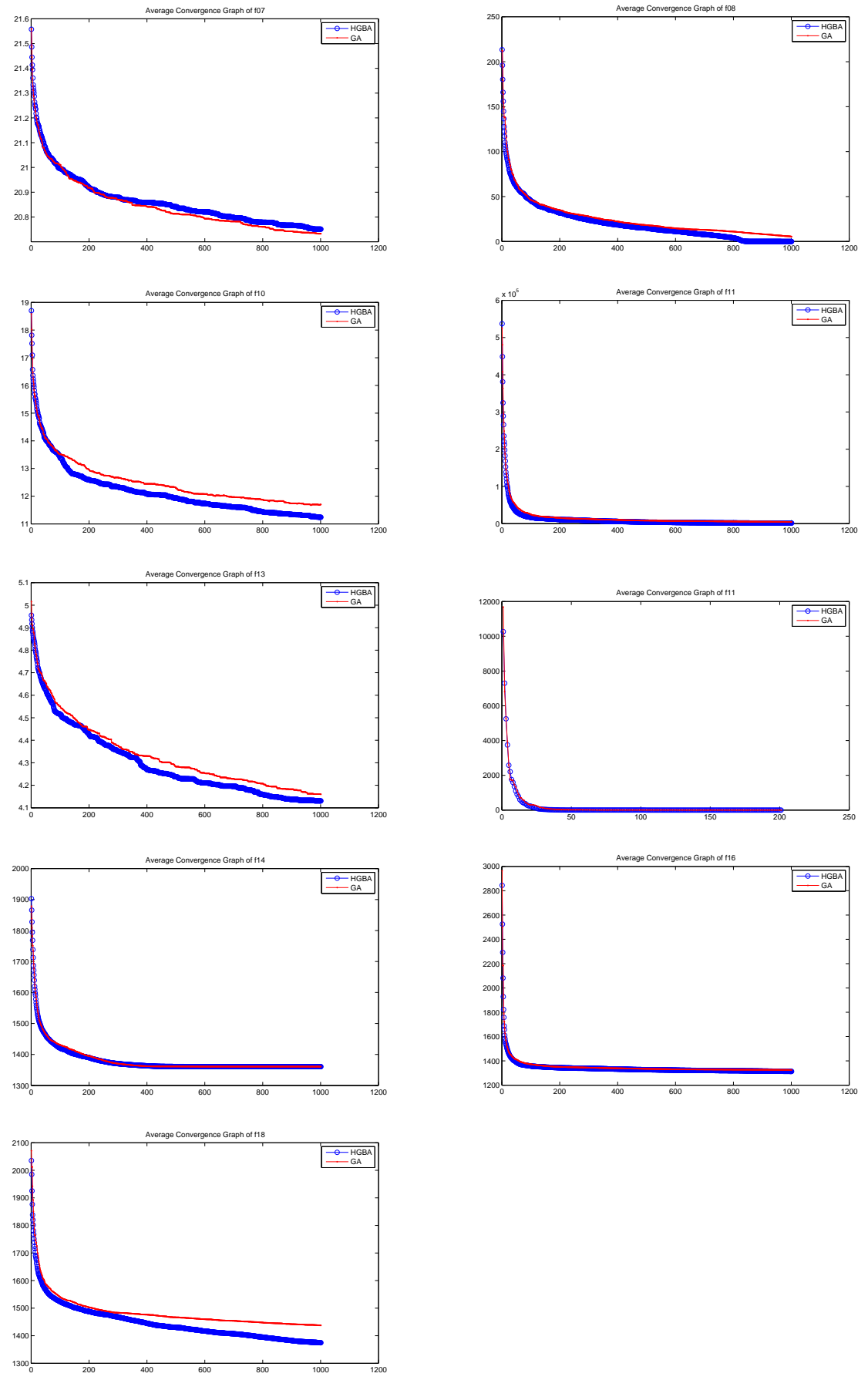

Figure 2. The convergence graph displayed by HGBA versus GA for CEC'05 [32] with thirty variables. 


\section{Acknowledgements}

This Research study is supported by National Research Program for Universities under the Project No 5892. The authors are also wish to acknowledge the help and corporation of the Hybrid Metaheuristic Computing Research Group King Khalid University (KKU), Kingdom of Saudi Arabia to make it possible this research contribution.

\section{References}

[1] S. Cagnoni, et al., Real-World Applications of Evolutionary Computing, Springer-Verlag Lecture Notes in Computer Science, Berlin, 2000.

[2] R. Chiong, Th. Weise, Z. Michalewicz (Editors), Variants of Evolutionary Algorithms for Real-World Applications, Springer, 2012, ISBN 3642234232

[3] Floudas, Christodoulos A., Panos M. Pardalos, Claire Adjiman, William R. Esposito, Zeynep H. Gums, Stephen T. Harding, John L. Klepeis, Clifford A. Meyer, and Carl A. Schweiger, Handbook of test problems in local and global optimization, Vol. 33. Springer Science \& Business Media, 2013.

[4] Wenyu Sun and Ya-Xiang Yua, Optimization Theory and Methods: Nonlinear Programming, Springer, ISBN 978-1441937650. pages, 541, 2010.

[5] Rituparna Datta and Kalyanmoy Deb,Evolutionary Constrained Optimization,Infosys Science Foundation Series in Applied Sciences and Engineering,ISSN: 2363-4995, Springer, 2015.

[6] S.H. Chen, J. Wu, and Y.D. Chen Interval optimization for uncertain structures, Finite Elements in Analysis and Design 40 (11), 1379-1398, 2004.

[7] MarcoCavazzuti, Optimization Methods: From Theory to Design Scientific and Technological Aspects in Mechanics, Springer Berlin Heidelber,2013.

[8] E. L. Lawler and D. E. Wood, Branch-and-Bound Methods: A Survey, Journal Operation Research, 14, 4, 699-719,Institute for Operations Research and the Management Sciences (INFORMS), Linthicum, Maryland, USA, 1996.

[9] R. Vetschera, A general branch-and-bound algorithm for fair division problems, Journal Computers and Operations Research, 37(12), 2121-2130, December, 2010.

[10] M. Veltman, Algebraic techniques, Computer Physics Communications, 3, 75-78, September, 1972.

[11] J.A Nelder and R.Mead, A simplex method for function minimization, The computer journal, 7(4), 308-313, 1965.

[12] S. M. Goldfeld, R. E. Quandt andH. F. Trotter , Maximization by quadratic hill-climbing. Econometrica: Journal of the Econometric Society,34(3), 541-551, 1966.

[13] S.Abbasbandy, Improving Newton-Raphson method for nonlinear equations by modified Adomian decomposition method, Applied Mathematics and Computation, 145(2), 887-893, 2003.

[14] X. Geng, J. Xu, J. Xiao, and L. Pan, A simple simulated annealing algorithm for the maximum clique problem, Information Sciences, 177, 22, 5064-5071, 2007.

[15] A. Eidehall and L. Petersson, Threat assessment for general road scenes using Monte Carlo sampling, IEEE Intelligent Transportation Systems Conference, 2006.

[16] M. Lin and J. Wawrzynek,Improving FPGA placement with dynamically adaptive stochastic tunneling, IEEE Transactions on Computer-Aided Design of Integrated Circuits and Systems, 29, 12, 1858-1869, 2010.

[17] J. Machta, Strengths and weaknesses of parallel tempering, Physical Review E- Statistical, Nonlinear, and Soft Matter Physics, 80, 5, 2009.

[18] A. Engelbrecht, Computational Intelligence- An Introduction $2^{\text {nd }}$ edition, Wiley, 2007.

[19] C. Pan, C. Xu, and G. Li, Differential evolutionary strategies for global optimization, Shenzhen Daxue Xuebao (Ligong Ban), Journal of Shenzhen University Science and Engineering, 25, 2, 211-215, 2008.

[20] B. Blaha and D. Wunsch, Evolutionary programming to optimize an assembly program, Proceedings of the 2002 Congress on Evolutionary Computation. CEC-02, 2, 1901-1903. 
[21] Fogel, L.J., Owens, A.J., Walsh, M.J., Artificial Intelligence through Simulated Evolution, John Wiley, 1966.

[22] Fogel, L.J., Intelligence through Simulated Evolution: Forty Years of Evolutionary Programming, John Wiley, 1999.

[23] J. Kennedy and R. Eberhart, Particle swarm optimization, in Proceedings of the IEEE International Conference on Neural Networks, 4, 1942-1948,1995 .

[24] J. Yu, L. Xi and S. Wang, An improved particle swarm optimization for evolving feedforward artificial neural networks, Neural Processing Letters, vol. 26, no. 3, pp. 217-231, 2007.

[25] C. Wu, Ant colony multilevel path optimize tactic based on information consistence optimize, 2010 International Conference on Computer Application and System Modeling (ICCASM 2010), 1, 533-536, November, 2010.

[26] R. Storn and K. Price,Differential Evolution: A Simple and Efficient Heuristic for Global Optimization over Continuous Spaces, Journal of Global Optimization, 11(4), 341-359, 1997.

[27] Ajith Abraham, Crina Grosan and Hisao Ishibuchi,Hybrid Evolutionary Algorithms, Studies in Computational Intelligence, Springer, 2007.

[28] X. Li, M. R.Bonyadi, Z.Michalewicz and L. Barone, A Hybrid Evolutionary Algorithm for Wheat Blending Problem, The Scientific World Journal, 2014.

[29] L.Zhang, L.Liu, XS Yang and Y.Dai, A novel hybrid firefly algorithm for global optimization, PloS one,11(9), 2016.

[30] Roger Fletcher, Practical methods of optimization, New York: John Wiley \& Sons, 1987.

[31] P.Venkataraman, Applied Optimization with Matlab programming, John Wiley \& Sons, New York, 2002.

[32] P. N. Suganthan, N. Hansen, J. J. Liang, K. Deb, Y.-P. Chen, A. Auger and S. Tiwari, Problem Definitions and Evaluation Criteria for the CEC 2005 Special Session on RealParameter Optimization, Technical Report, Nanyang Technological University, Singapore and KanGAL Report No:2005005, IIT Kanpur, India.

[33] J. Holland, Adaptation in Natural and Artificial Systems: The University of Michigan, 1975.

[34] K. De Jong, An analysis of the behavior of a class of genetic adaptive systems, Doctoral Dissertation. Ann Arbor: The University of Michigan, 1975.

[35] Wang G-G, Guo L, Gandomi AH, Hao G-S, Wang H, Chaotic krill herd algorithm, Information Science, 274, 17-34, 2014.

[36] Wang G-G, Gandomi AH, Alavi AH, Stud krill herd algorithm, Neurocomputing, 128, 363$370,2014$.

[37] Wang G, Guo L, Wang H, Duan H, Liu L, Li J, Incorporating mutation scheme into krill herd algorithm for global numerical optimization, Neural Comput Appl 24(3-4):853-871, 2014.

[38] Wang G-G, Deb S, Cui Z, Monarch butterfly optimization, Neural Comput Applcation, 2015.

[39] Wang G-G, Deb S, Coelho LdS, Earthworm optimization algorithm: a bio-inspired metaheuristic algorithm for global optimization problems, Int $\mathrm{J}$ of Bio-Inspired Computation, 2015.

[40] Sulaiman, Muhammad, Salhi, Abdellah, Mashwani, Wali Khan, and Rashidi, Muhammad M, A Novel Plant Propagation Algorithm: Modifications and Implementation, Science International, 28 (1), 201-209, 2016.

[41] Sulaiman, Muhammad, and Salhi, Abdellah, A Seed-based Plant Propagation Algorithm: The Feeding Station Model, The Scientific World Journal, 1-16, 2015

[42] Sulaiman, Muhammad, Salhi, Abdellah, Selamoglu, Birsen Irem, Bahaaldin Kirikchi, Omar, A Plant Propagation Algorithm for Constrained Engineering Optimisation Problems, Mathematical problems in engineering, 1-10, 2014.

[43] Abdellah Salhi and Eric S. Fraga, Nature-Inspired Optimisation Approaches and the New Plant Propagation Algorithm, Proceedings of the ICeMATH2011, K2-1 to K2-8.

[44] A.E. Eiben and J.E. Smith, Introduction to Evolutionary Computing, Springer, Natural Computing Series, 2003.

[45] Wang G-G, Gandomi AH, Alavi AH, Hao G-S, Hybrid krill herd algorithm with differential evolution for global numerical optimization, Neural Comput Appl, 25(2):297-308, 2014. 
[46] Wang G-G, Gandomi AH, Zhao X, Chu HE, Hybridizing harmony search algorithm with cuckoo search for global numerical optimization, Soft Computing, 20(1):273-285, 2016.

[47] Muhammad Asim, Wali Khan Mashwani and M.A.Jan, Hybrid Genetic Firefly Algorithm for Global Optimization Problems, Sindh University Research Journal, 49(3), 2017.

[48] Muhammad Asim, Wali Khan Mashwani, Muhammad Asif Jan and Javed Ali, Derivative Based Hybrid Genetic Algorithm: A Preliminary Experimental Results, Punjab University Journal of Mathematics, Vol. 49(2) (2017) pp. 89-99.

[49] Khug Alam,Wali Khan Mashwani and Muhammad Asim, Hybrid Biography Based Optimization Algorithm for Optimization Problems, Gomal University Journal of Research, Vol 33, issue1, pp:134-142, 2017.

[50] Hamza Wazir, Muhammad Asif Jan, Wali Khan Mashwani and Tayyaba Shah, A Penalty Function Based Differential Evolution Algorithm for Constrained Optimization, The Nucleus Journal, Vol 53, No. 1 (2016) 155-161.

[51] Habib Shah, Nasser Tairan, Wali Khan Mashwani, Abdulrahman Ahmad Al-Sewari, Muhammad Asif Jan, Gran Badshah, Hybrid Global Crossover Bees Algorithm for Solving Boolean Function Classification Task, International Conference on Intelligent Computing ICIC (3) 2017: 467-478

[52] D. E. Goldberg, Genetic Algorithms in Search, Optimization, and Machine Learning: Addison-Wesley, 1989.

[53] J. H. Holland, Adaptation in Natural and Artificial Systems: an Introductory analysis with Applications to Biology, Control, and Artificial Intelligence, MIT Press, 1992.

[54] Melanie Mitchell, An Introduction to Genetic Algorithms, MIT Press Cambridge, MA, USA, 1998.

[55] Jyoti Sharma and Ravi Shankar Singhal,Genetic Algorithm and Hybrid Genetic Algorithm for Space Allocation Problems-A Review, International Journal of Computer Applications, 95(4), 2014.

[56] Tarek A. El-Mihoub, Adrian A. Hopgood, Lars Nolle and Alan Battersby, Hybrid Genetic Algorithms: A Review, Engineering Letters, 13(2), 124-137, 2006.

[57] Rashida Adeeb Khanum, Nasser Mansoor Tairan, Muhammad Asif Janan d Wali Khan Mashwani, Hybridization of Adaptive Differential Evolution with an Expensive Local Search Method, Journal of Optimization, Volume 2016 (2016), Article ID 3260940, 14 pages

[58] R. Farmani and J. A. Wright,Self-Adaptive Fitness Formulation for Constrained Optimization, IEEE Transactions on Evolutionary Computation,7, 445-455, 2003.

[59] Homaifar, A., Lai, S.H.Y. and Qi, X , Constrained optimization via genetic algorithms, Simulation, 62, 242-254, 1994.

[60] H. Liu, Z. Cai, and Y. Wang, Hybridizing particle swarm optimization with differential evolution for constrained numerical and engineering optimization, Applied Soft Computing, $10(2), 629-640,2010$.

[61] Tayaba Shah, Muhammad Asif Jan, Wali Kahan Mashwani and Hamza Wazir, Adaptive differential evalution for constrained optimization problems, Science Int.(Lahore), 3(28) 4104-4108, 2016.

[62] Wali Khan Mashwani, Abdellah Salhi, Muhammad Asif. Jan, Muhammad Sulaiman, R.A. Khanum and Abdulmohsen Algarni,Evolutionary Algorithms Based on Decomposition and Indicator Functions: State-of-the-art Survey, International Journal of Advanced Computer Science and Applications, 7(2), 583-593, 2016

[63] J. J. Liang, T. P. Runarsson, E. Mezura-Montes, M. Clerc, P. N. Suganthan1, C. A. Coello Coello and K. Deb, Problem definitions and evaluation criteria for the CEC 2006 special session on constrained real-parameter optimization, Technical Report, Nanyang Technological University, Singapore, 2006. 